\title{
DGCR8-mediated disruption of miRNA biogenesis induces cellular senescence in primary fibroblasts
}

Daniel Gómez-Cabello, ${ }^{1 *,+}$ Isabel Adrados, ${ }^{1 *}$ David Gamarra, ${ }^{1 \neq}$ Hikaru Kobayashi, ${ }^{1}$ Yoshihiro Takatsu, ${ }^{2}$ Kyoko Takatsu, ${ }^{2}$ Jesús $\mathrm{Gil}^{2}$ and Ignacio Palmero'

${ }^{1}$ Instituto de Investigaciones Biomédicas 'Alberto Sols' CSIC-UAM, Madrid, Spain

${ }^{2}$ Cell Proliferation Group, MRC Clinical Sciences Centre, Imperial College London, London, UK

\section{Summary}

The regulation of gene expression by microRNAs (miRNAs) is critical for normal development and physiology. Conversely, miRNA function is frequently impaired in cancer, and other pathologies, either by aberrant expression of individual miRNAs or dysregulation of miRNA synthesis. Here, we have investigated the impact of global disruption of miRNA biogenesis in primary fibroblasts of human or murine origin, through the knockdown of DGCR8, an essential mediator of the synthesis of canonical miRNAs. We find that the inactivation of DGCR8 in these cells results in a dramatic antiproliferative response, with the acquisition of a senescent phenotype. Senescence triggered by DGCR8 loss is accompanied by the upregulation of the cell-cycle inhibitor p21CIP1. We further show that a subset of senescence-associated miRNAs with the potential to target p21CIP1 is downregulated during DGCR8-mediated senescence. Interestingly, the antiproliferative response to miRNA biogenesis disruption is retained in human tumor cells, irrespective of p53 status. In summary, our results show that defective synthesis of canonical microRNAs results in cell-cycle arrest and cellular senescence in primary fibroblasts mediated by specific miRNAs, and thus identify global miRNA disruption as a novel senescence trigger.

Key words: DGCR8; fibroblasts; microRNA; p21CIP1; p53; senescence.

\section{Introduction}

Tumors arise as a consequence of the malfunction of critical regulators of normal cellular homeostasis, which results in aberrant proliferation and other tumor-associated traits. Genetic studies in cancer have long focused on genes that code for proteins. However, in recent years, it has become increasingly clear the essential role of regulatory noncoding RNAs, such as microRNAs (miRNAs) or long noncoding RNAs (IncRNAs) in

\section{Correspondence}

Ignacio Palmero, Instituto de Investigaciones Biomédicas 'Alberto Sols' CSIC-UAM. Arturo Duperier, 4, Madrid 28029, Spain. Tel.: (+34) 915854491; fax: (+34) 915854401; e-mail: ipalmero@iib.uam.es

*These authors contributed equally.

†Present address: Centro Andaluz de Biología Molecular y Medicina Regenerativa (CABIMER), Seville, Spain

"Present address: Centro de Biología Molecular 'Severo Ochoa', Madrid, Spain

Accepted for publication 09 June 2013 cancer and other human pathologies. These regulatory RNAs play a key role in the control of cellular functions critically altered in tumor formation and progression, and accordingly their deregulation is an almost universal feature of tumors (Esteller, 2011; Lujambio \& Lowe, 2012). MicroRNAs are small noncoding RNAs (18-25 nucleotides) that negatively regulate their target genes, primarily through RNA destabilization and inhibition of translation. The synthesis of mature microRNAs involves a series of coordinated steps in the nucleus and cytoplasm (Winter et al., 2009). A primary transcript (pri-miRNA) is initially generated by RNA polymerase II from independent miRNA units or clusters located in the introns of protein-coding genes, or other genomic locations. The primary transcript is processed in the nucleus by a complex formed by the ribonuclease Drosha, and the auxiliary protein DGCR8 (also known as Pasha) to generate 60- to 70-bp-long RNA hairpins, known as pre-miRNA. An alternative, noncanonical pathway leads to the generation of pre-miRNA molecules from splicing of intron-encoded precursors, mirtrons, without the participation of the Drosha-DGCR8 complex. pre-miRNAs are subsequently exported to cytoplasm where they are further processed by Dicer and loaded into the RNA-induced silencing complex (RISC) to produce destabilization and translational inhibition of target genes (Guo et al., 2010). Aberrant miRNA expression is a common trend of human cancers. There are many examples of individual miRNAs with deregulated expression in tumors, which play both positive and negative roles in tumor formation or progression (Esteller, 2011; Lujambio \& Lowe, 2012). In addition, global regulation of miRNA biogenesis can also be altered during tumorigenesis, as shown by the deregulation in tumors of key miRNA-processing factors (Esteller, 2011; Lujambio \& Lowe, 2012). Cellular senescence is increasingly recognized as an essential tumor-suppressive mechanism that restrains uncontrolled proliferation of cells harboring potentially oncogenic alterations. Senescence can be induced by a large series of alterations in normal cellular homeostasis associated with tumorigenesis, including activation of oncogenes, DNA-damage, telomere dysfunction, or inactivation of tumor suppressor genes (Serrano et al., 1997; Collado \& Serrano, 2010; Kuilman et al., 2010). Here, we set to study the global impact of miRNA biogenesis in primary cells, using the specific knockdown of DGCR8, a key regulator of canonical miRNAs synthesis. We find that disrupted microRNA synthesis results in a senescence-like antiproliferative response in primary fibroblasts, as a consequence of the deregulation of key miRNAs linked to the cell-cycle machinery, and this response is essentially retained in tumor cell lines.

\section{Results}

\section{Disruption of miRNA biogenesis blocks proliferation in primary cells}

To study the global role of miRNA-mediated gene regulation in nontransformed, primary cells, we inhibited miRNA biogenesis by stable silencing of key regulators of this pathway in primary fibroblasts. First, early-passage IMR90 human primary fibroblasts were infected with vectors expressing shRNAs against DGCR8, which efficiently reduced endogenous DGCR8 mRNA and protein levels (Fig. 1A, left and Fig. S1A). Inactivation of DGCR8 dramatically reduced the proliferation 
(A)
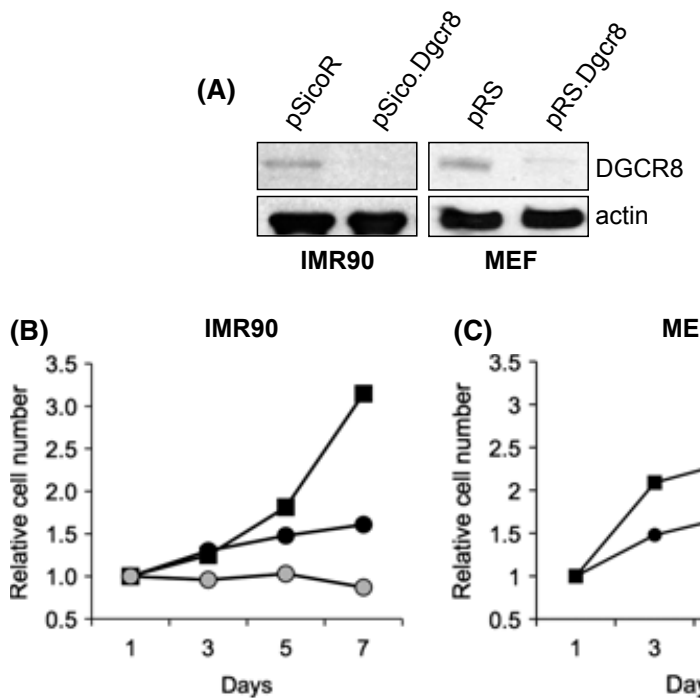

(C)
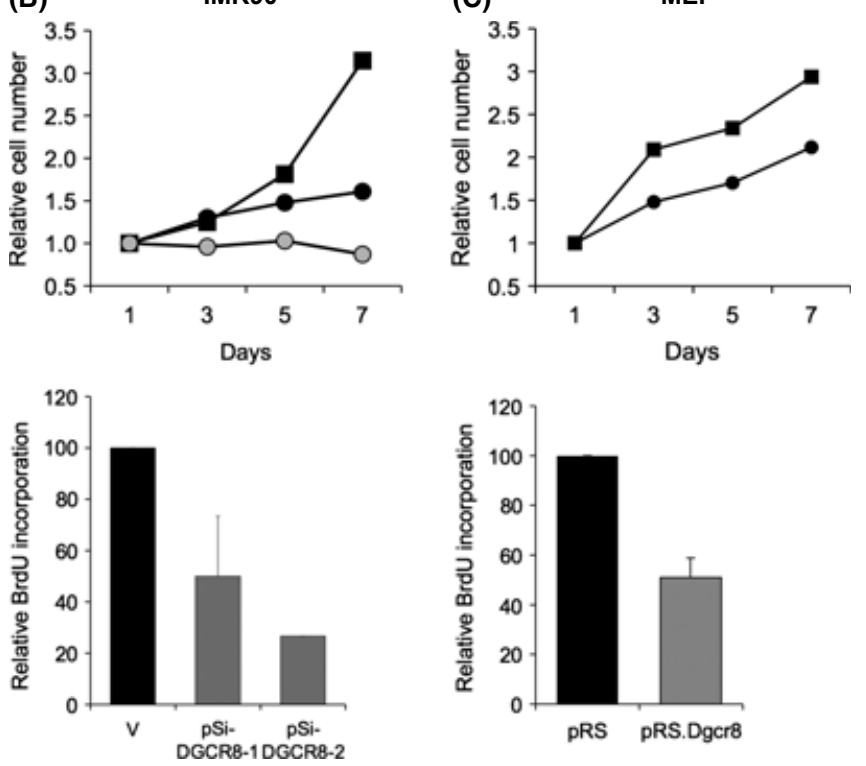

(D)

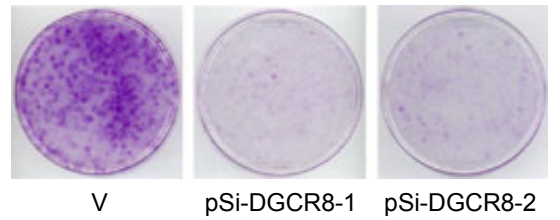

Fig. 1 Antiproliferative effect of abrogation of DGCR8 in fibroblasts. (A) Western blot showing silencing efficiency of sh-DGCR8 vectors. (B) Growth curve, top, and BrdU incorporation rate (bottom) of IMR-90 human fibroblasts infected with two different sh-DGCR8 vectors (dots in top panel) or empty vector (black squares). (C) Growth curve, top, and BrdU incorporation rate (bottom) of wild-type mouse embryo fibroblasts infected with a sh-DGCR8 vector or empty vector. (D) Crystal violet staining of IMR-90 human fibroblasts retrovirally infected with two shDGCR8 vectors or empty vector. $(B, C)$ bottom panels show the average and SD of at least three independent experiments.

rate of these cells, as shown by their cummulative growth curves and their rate of BrdU incorporation (Fig. 1B). The antiproliferative effect was also evident in long-term growth assays, or in studies using low cell density (Figs 1D and S1B). Consistent results were obtained using two independent shRNAs targeting DGCR8 (Fig. 1B). Similar results were obtained with shRNAs against Drosha and Dicer, two additional key regulators of miRNA biogenesis (Fig. S1A,C,D) further confirming that the phenotype observed was triggered by disruption of miRNA biogenesis. To test the generality of these observations, we also inactivated DGCR8 in early-passage wild-type mouse embryo fibroblasts (MEF) using a retroviral shRNA vector (Fig. 1A, right). Similar to human fibroblasts, stable silencing of DGCR8 in MEFs resulted in reduced BrdU incorporation and proliferation rate, relative to control MEFs, although the effect was less dramatic than that seen with human fibroblasts (Fig. 1C). This

set of results indicates that global disruption of miRNA biogenesis blocks proliferation of primary fibroblasts.

\section{Induction of senescence in DGCR8-deficient fibroblasts}

Having shown that disruption of miRNA biogenesis in fibroblasts triggers an antiproliferative response, we wanted to characterize the mechanism responsible for this arrest in shDGCR8 fibroblasts. During the course of our experiments, we noticed that ShDGCR8 IMR90 fibroblasts underwent changes in morphology that were reminiscent of those of senescent fibroblasts. Cellular senescence is an antiproliferative response and tumor-suppressive barrier, which can be triggered by a variety of potentially oncogenic changes in cellular homeostasis (Collado \& Serrano, 2010). We hypothesized that the disruption of miRNA biogenesis could activate senescence, thus explaining the proliferative defect observed. To test this hypothesis, we assayed several wellestablished markers of the senescent phenotype. As mentioned, IMR90 cells expressing shDGCR8 acquired a characteristic flat morphology, typical of senescent fibroblasts, in contrast to the long elongated shape of nonsenescent human fibroblasts. Similarly, shDGCR8 IMR90 cells also showed a significant increase in the number of cells with senescentassociated beta-galactosidase (SA-Beta Gal) activity (Fig. 2A) as well as nuclei with SAHFs (senescence-associated heterochromatin foci), regions of facultative heterochromatin characteristic of senescent cells (Fig. 2B). Taken together, these results indicate that the depletion of DGCR8 leads to premature senescence in IMR90 human fibroblasts. Prompted by these results, we also studied whether silencing of DGCR8 could induce a senescent phenotype in MEFs, a cell type where we had also observed reduced proliferation by shDGCR8. As shown in Figure 2C, DGCR8deficient wild-type MEFs also acquired a senescent phenotype, as indicated by a clear increase in cells positive for SA-Beta Gal activity and with flattened morphology. To identify possible mediators of the phenotype of DGCR8-deficient cells, we carried out Western Blot analysis of key regulators of cell cycle and senescence in MEFs and IMR90 fibroblasts. Inactivation of DGCR8 in human and murine fibroblasts caused a modest, but reproducible increase in the protein levels of the cell-cycle inhibitor p21CIP1 (Figs 2D and E). Also, a 3-fold increase in p21CIP1 transcript (Fig. 2F) and increased number of p21CIP1-positive cells by immunofluorescence (Fig. 2G) was observed in shDGCR8 human fibroblasts. Of note, p21CIP1 is frequently upregulated and contributes to cell-cycle arrest during senescence (Brown et al., 1997), and it is directly regulated by miRNAs (Ivanovska et al., 2008; Wu et al., 2010). We did not observe significant changes in p53 or p16 protein levels in either cell type after DGCR8 silencing (Fig. 2D,E and data not shown).

\section{Mediators of DGCR8-induced senescence}

To characterize further, the mechanism behind the induction of senescence by miRNA disruption, we tried to identify potential mediators of the response. For human fibroblasts, we focused on the p53 and p16/ $\mathrm{Rb}$ pathways, key tumor suppressors, which play a critical role in the implementation of senescence in this cell type (Serrano et al., 1997; Collado \& Serrano, 2010). To this end, we combined ShDGCR8 with specific shRNAs against p53 or Rb, to achieve stable independent inactivation of each pathway. shRNAs against p16INK4A were also used in some experiments to target the $\mathrm{p} 16 / \mathrm{Rb}$ pathway, giving undistinguishable results (data not shown). Efficient silencing of each protein was achieved, while endogenous p53 or Rb protein levels were not significantly affected by DGCR8 downregulation (Fig. S2A). IMR90 cells deficient for $\mathrm{Rb}$ or $\mathrm{p} 53$ drastically reduced proliferation upon DGCR8 
(A)

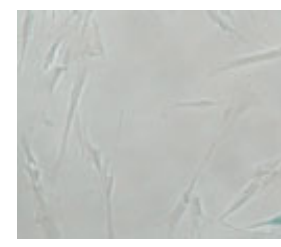

(B)
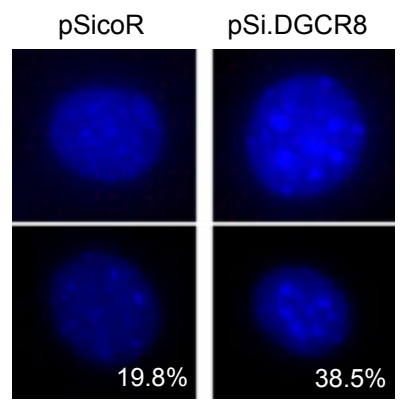

(C) pRS
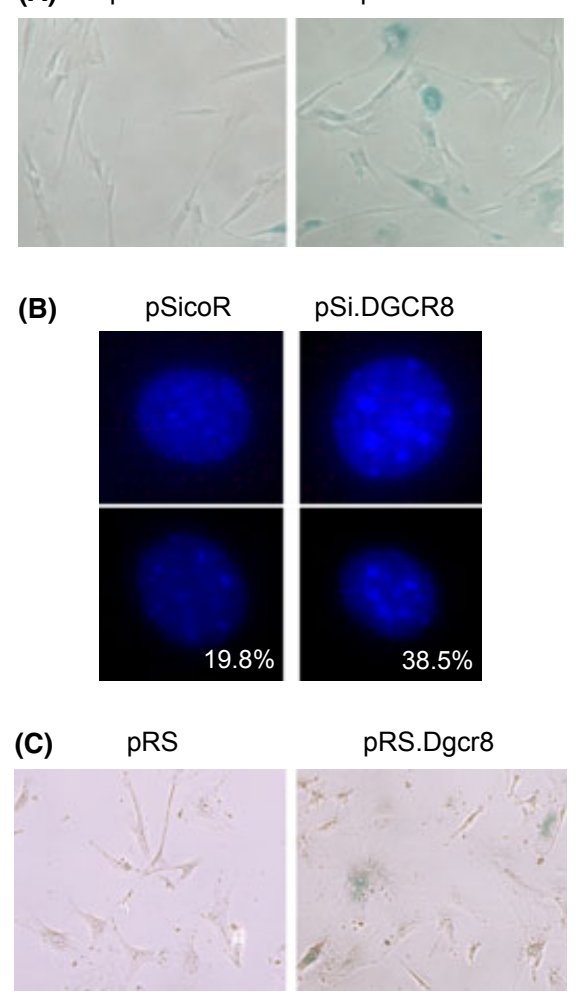

$38.5 \%$
(D)

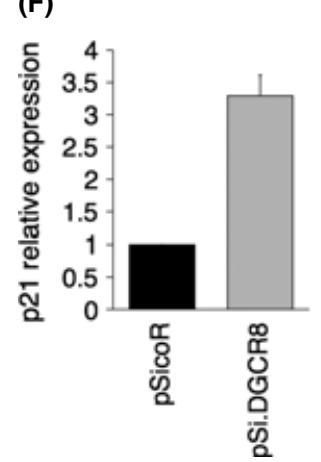

(E)

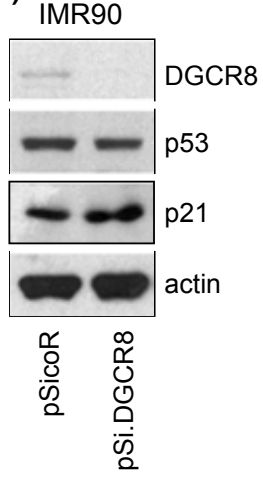

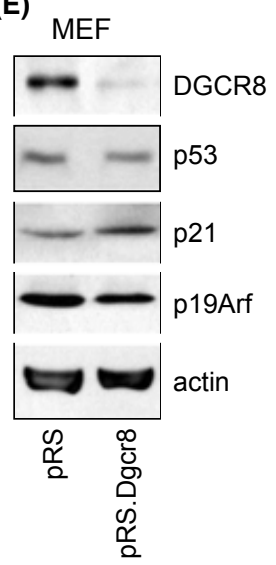

(G)

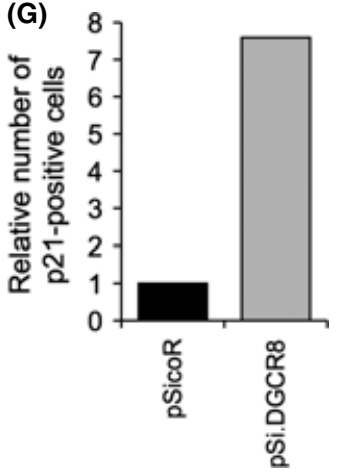

Fig. 2 Induction of senescence markers in sh-DGCR8 fibroblasts. (A,B) SA-Beta Gal and SAHFs in IMR-90 fibroblasts expressing sh-DGCR8 or empty vector. (B) two representative images and the percentage of SAHF-positive cells are indicated. (C) SA-Beta Gal staining in mouse embryo fibroblasts expressing sh-Dgcr8 or empty vector. $(D, E)$ Western blot analysis of the indicated proteins in human, (D) and murine, (E) fibroblasts expressing sh-DGCR8 vectors or empty vector controls. (F) QPCR analysis of transcript levels for p21CIP1 in human IMR-90 fibroblasts. The average and standard deviation from three experiments is shown. (G) Number of IMR90 cells positive for p21CIP1 by immunofluorescence.

inactivation, to a similar extent as parental IMR90 fibroblasts, as measured by BrdU incorporation rate. Similarly, shp53 or shRb IMR90 fibroblasts expressing shDGCR8 acquired senescent markers, such as enlarged morphology, SA-Beta Gal activity or SAHFs to an extent comparable to control fibroblasts (Fig. 3A-D). Collectively, these results indicate that depletion of DGCR8 can induce a senescent phenotype in human primary fibroblasts that is mainly p53 and Rb independent. Similar analyses were performed in mouse fibroblasts, using p53-knockout and p21-knockout MEFs, the latter due to the increase in p21 observed in response to DGCR8 deficiency. We confirmed that high efficiency of DGCR8 silencing was obtained in all the genotypes (Fig. 3E). Growth curves and BrdU incorporation assays revealed that p53-knockout MEFs were less responsive to DGCR8 silencing than wild-type counterparts. Instead, p21 knockout MEFs showed a reduction in proliferation comparable to wild-type MEFs (Fig. 3F,G). These results with human and mouse fibroblasts are consistent with the notion that p53 disruption suffices to bypass senescence in murine fibroblasts, whereas simultaneous inactivation of both the $\mathrm{Rb}$ and $\mathrm{p} 53$ pathways is generally required for that effect in human fibroblasts (Serrano et al., 1997).

\section{Mechanism of senescence activation in DGCR8-deficient primary cells}

To distinguish which could be the mechanism linking DGCR8-deficiency to senescence, we considered several hypotheses. First, we asked if the phenotype could be caused by Ras-induced senescence. Sustained mitogenic signaling due to activated Ras oncogene is a well-characterized trigger for cellular senescence in fibroblasts and other cell types (Serrano et al., 1997). In addition, it has been shown that Ras expression is regulated by the let-7 miRNA family, providing a direct link between miRNA function and Ras activity (Johnson et al., 2005). To test this hypothesis, we measured Ras levels and phosphorylation of the downstream Ras effectors ERK1/2 in ShDGCR8 fibroblasts. Although we observed a transient increase in Ras protein levels and ERK phosphorylation in some experiments with shDGCR8 wild-type MEFs, we failed to detect consistent activation of the Ras pathway (Fig. S2B). p19Arf accumulates dramatically in MEFs upon oncogenic Ras expression or other pro-senescent stimuli (Zindy et al., 1997; Palmero et al., 1998). We found that p19Arf was unchanged or even downregulated in shDGCR8 MEFs in our experiments (Figs 2E and $\mathrm{S} 2 \mathrm{~B})$, arguing against the occurrence of canonical oncogene-induced senescence in these cells. In addition, shDGCR8 MEFs that lacked p21 or p53 did not show clear changes in Ras protein or its signaling pathway, with similar p19Arf levels. On the other hand, DGCR8deficient IMR90 cells did not show significant changes in the Ras pathway or induction of the p16INK4A protein (the INK4AVARF product more commonly activated during senescence in this cell type, data not shown). As an alternative explanation for the senescent phenotype, we also investigated if suppression of DGCR8 could lead to DNA damage, a potential trigger of senescence (Di Micco et al., 2006). We did not 


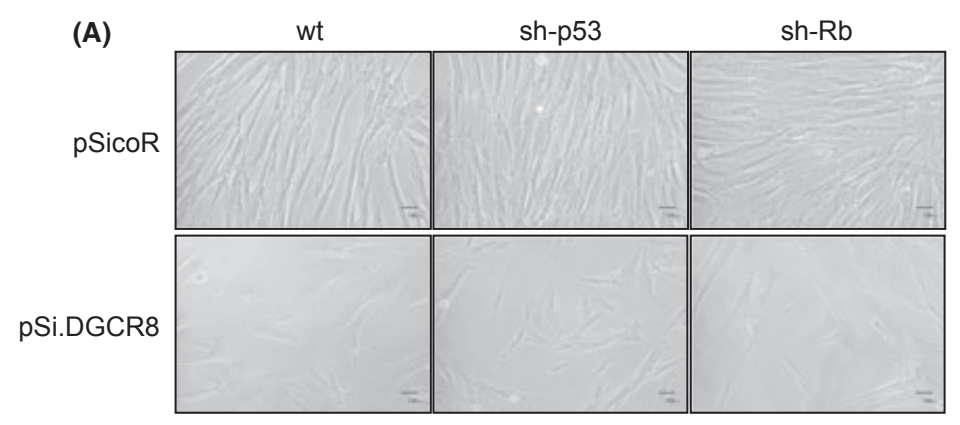

(B)

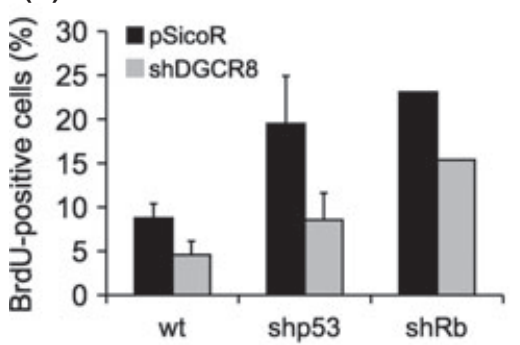

(E)
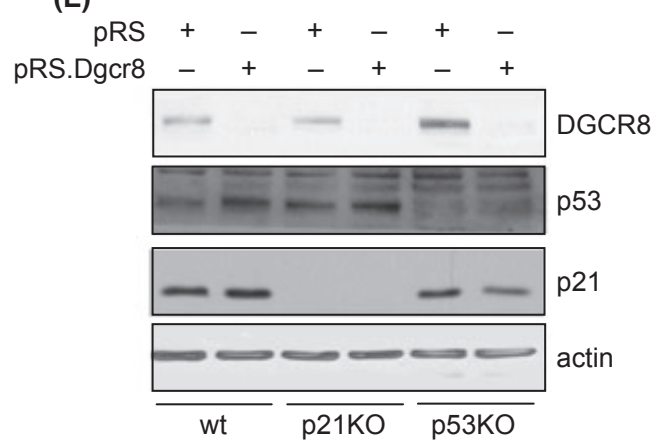

(C)

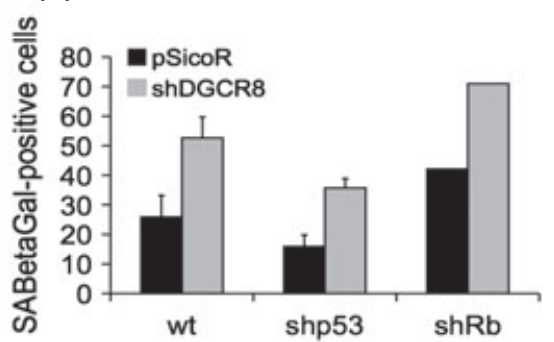

(F)

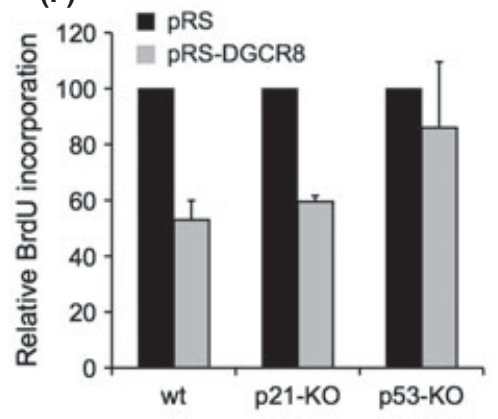

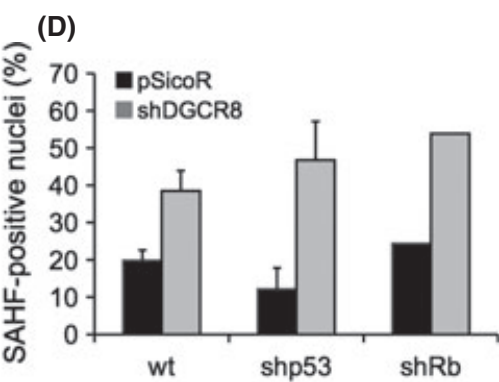

(G)

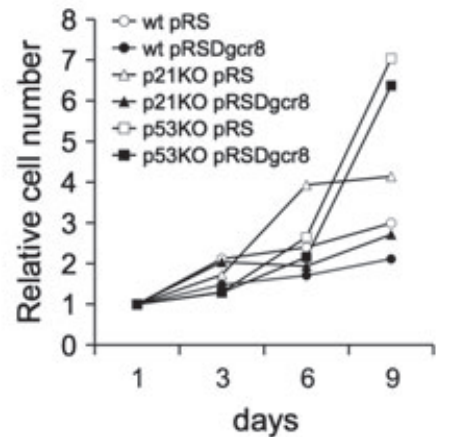

Fig. 3 Molecular mediators of DGCR8-induced senescence. (A) Representative micrographs showing senescent morphology in IMR-90 fibroblasts, expressing the indicated combinations of sh-RNA vectors. (B,C,D) BrdU incorporation rates (B), percentage of SA-BetaGal positive cells (C), or SAHF-positive nuclei (D), of IMR-90 cells expressing the indicated combinations of sh-RNA vectors, 6 days after the end of drug selection. The average and standard deviation from three experiments is shown. (E) Western blot analysis of the indicated proteins in wild-type, p21CIP1-KO or p53-KO MEFs, expressing an sh-Dgcr8 vector or empty vector. (F, G) Relative

BrdU incorporation and growth curve. The average and standard deviation from three experiments is shown in (F). (G) shows data from one representative experiment.

observe significant changes in the DNA-damage marker gamma-H2AX, in shDGCR8 human fibroblasts of two different strains (Fig. S3B). Likewise, no gross changes were noticed in chromatin or nuclear structure after silencing of DGCR8 in murine or human fibroblasts (leaving aside appearance of SAHFs in IMR90, Fig. S3A). Collectively, these results suggest that activation of Ras-induced senescence or DNA damage cannot account for the arrest triggered by shDGCR8 in primary fibroblasts.

\section{Role of specific miRNAs in DGCR8-induced arrest}

Studies with mice genetically defective for Dgcr8 (Stark et al., 2008; Wang et al., 2008) have shown that, despite the general deregulation of miRNAs, the proliferation defects associated with loss of DGCR8 can be traced to a subset of individual miRNAs. In order to identify specific miRNAs that could be mediating the phenotype triggered by DGCR8dependent disruption of miRNA biogenesis, we analyzed the expression of individual miRNAs in IMR90 fibroblasts upon DGCR8 suppression. As a first step, we identified a set of miRNAs downregulated in IMR90 fibroblasts made senescent by tamoxifen-inducible activation of MEK (Fig. 4A). Interestingly, we noted a significant overlap between this set of senescence-regulated miRNAs and those associated to cell-cycle defects in DGCR8-null embryonic stem cells and early embryos (Wang et al., 2007, 2008). With this rationale, we focused on the miRNAs miR20a, miR-93 and miR-106a as potential mediators of DGCR8-induced senescence. The expression analysis of this subset of miRNAs in IMR90 fibroblasts showed that they were significantly downregulated during DGCR8-mediated arrest (Fig. 4B). All these miRNAs share a common seed sequence, and two potential binding sites for them were identified in the 3'UTR region of human p21CIP1 using two different prediction tools, Targetscan and Pictar, (Fig. S4, see also Ivanovska et al., 2008; Wang et al., 2008). Their ability to target the 3'UTR of the human p21CIP1 transcript was subsequently confirmed in a luciferase reporter assay (Fig. 4C). To test directly the participation of this set of miRNAs in 
(A)

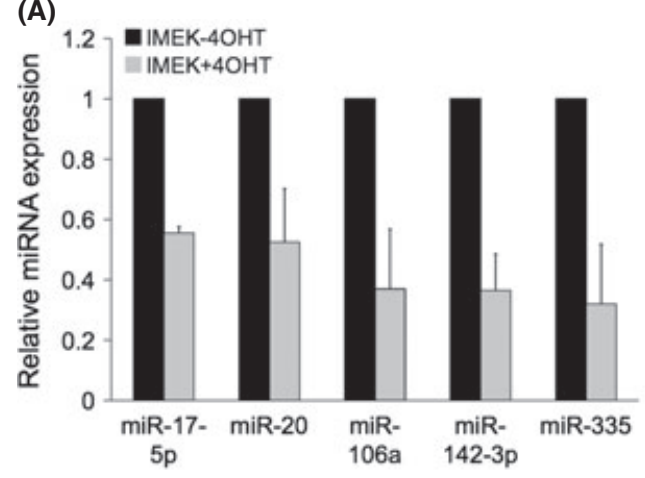

(C)

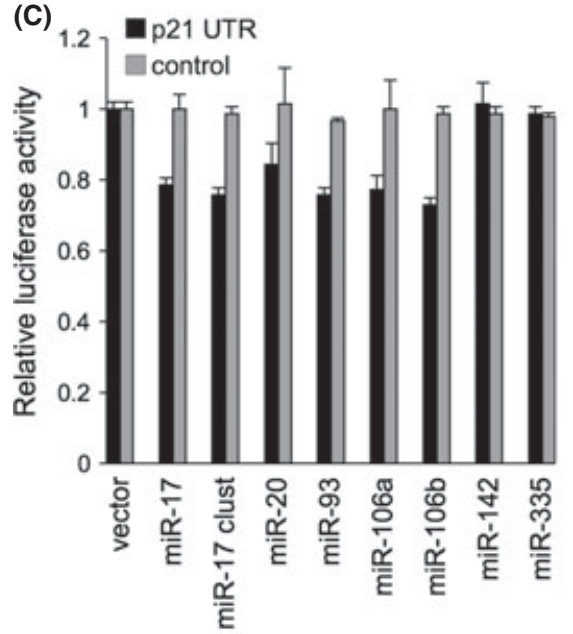

(B)

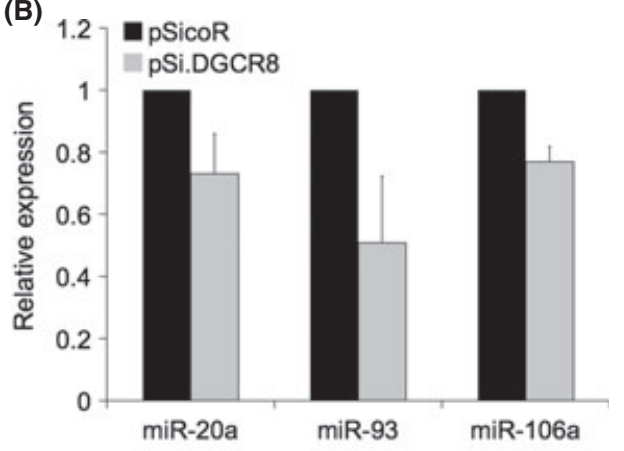

(D)

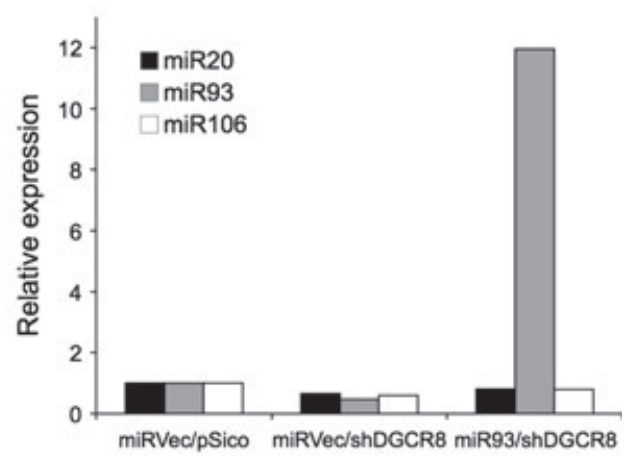

(E)

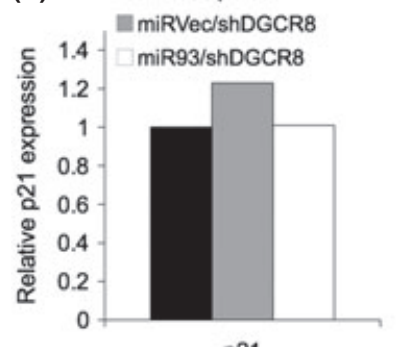

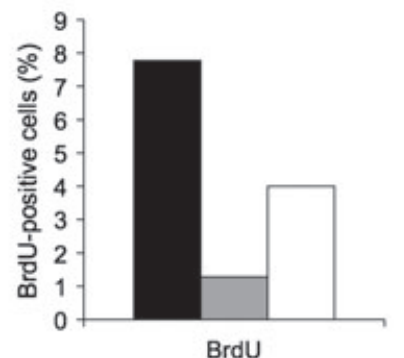

BrdU

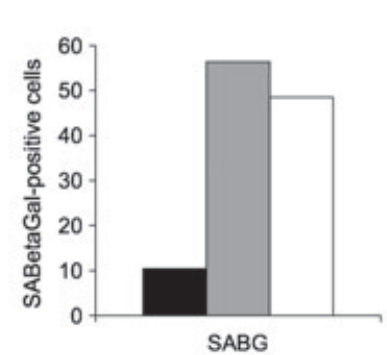

Fig. 4 Role of specific microRNAs. (A) QPCR of the indicated mature miRNAs in IMR-90 fibroblasts expressing a tamoxifen-inducible version of MEK (IMEK), with or without 4-OH-tamoxifen (4OHT). The transcript levels relative to untreated cells are shown. (B) QPCR of the indicated mature miRNAs in IMR-90 fibroblasts expressing sh-DGCR8, relative to vector-expressing cells. (C) Luciferase activity of p21CIP1 3'UTR luciferase reporter constructs, after cotransfection with the indicated miRNAs. The vector referred as 'miR-17 clust' encodes the polycistronic miR-17-92 cluster, which includes miR-17 and miR-20a, among other miRNAs. The average and standard deviation from two independent assays are shown. (D) QPCR of the indicated miRNAs in IMR-90 cells serially infected with vectors for shDGCR8 and ectopic miR-93, and corresponding empty vectors. (E) QPCR of p21CIP1 (left), BrdU incorporation (centre), and percentage of SA-BetaGal-positive cells (right) in IMR-90 cells expressing the indicated combinations of shRNA and ectopic miRNA vectors. $(D, E)$ show data from one representative experiment.

DGCR8-induced senescence, we expressed them ectopically in combination with silencing of DGCR8. Of the three miRNAs tested, we focused on miR-93, based on its degree of downregulation in shDGCR8 cells (Fig. 4B) and the level of overexpression achieved (Fig. 4D and data not shown). We found that ectopic miR-93 was able to bypass to different extents the effect of shDGCR8 in IMR90 cells as measured by p21 induction, BrdU incorporation and SA-Beta Gal activity (Fig. 4E). These results strongly suggest that this set of miRNAs are potential mediators of the senescent phenotype and p21 induction triggered by defective miRNA biogenesis.

\section{DGCR8 deficiency causes arrest in cancer cells}

Our findings show that a proficient miRNA-processing machinery is essential for normal proliferation in primary fibroblasts, and its disruption is sufficient to trigger cellular senescence. Based on these observations, we asked if experimental manipulation of miRNA biogenesis could also be exploited to block proliferation in tumor cells. To this end, DGCR8 was silenced in the human colorectal cancer HCT116 cell line (Fig. 5A). Similarly to human and mouse primary cells, DGCR8 depletion had a clear antiproliferative effect in this tumor cell line as shown by a reduction of 
colony numbers (Fig. 5D), and in cell number (Fig. S5A). Silencing of DGRC 8 also led to an increase in p21, at protein and mRNA levels (Fig. 5A, B). Similar results were obtained with additional human transformed or immortalized cell lines (Fig. S5B, see also Gomez-Cabello et al., 2010). Of note, the upregulation of $\mathrm{p} 21$ protein in human tumor or immortalized cells was more intense than that observed in primary fibroblasts (Figs $5 \mathrm{~A}$ and $\mathrm{S} 5 \mathrm{~B})$. Interestingly, DGCR8 silencing in these cells resulted in a significant decrease in expression of miR-20a, miR-93, and miR-106a, the same set of miRNAs deregulated in shDGCR8 human fibroblasts, (Fig. 5C). A p53-null derivative of the HCT116 cell line was also efficiently arrested by shDGCR8, indicating that, similar to primary human fibroblasts, the antiproliferative effect of shDGCR8 in tumor cell lines was retained in the absence of functional p53 (Figs 5A,D and S5A). However, in contrast to primary fibroblasts, the DGCR8-mediated arrest in HCT-116 cells was not accompanied by a clear induction of classical senescence markers, such as SA-Beta Gal staining, SAHF formation or morphology changes (data not shown).

\section{Discussion}

In this report, we have shown that the inactivation of DGCR8, an essential regulator of biogenesis of canonical miRNAs, results in cell-cycle arrest in primary fibroblasts, with the characteristic features of cellular senescence. Our observations are in line with extensive genetic evidence in mouse models with loss of function of miRNA regulators, which supports the notion that disruption of miRNA production is detrimental for normal development and proliferation. Thus, targeted disruption of the Dgcr8
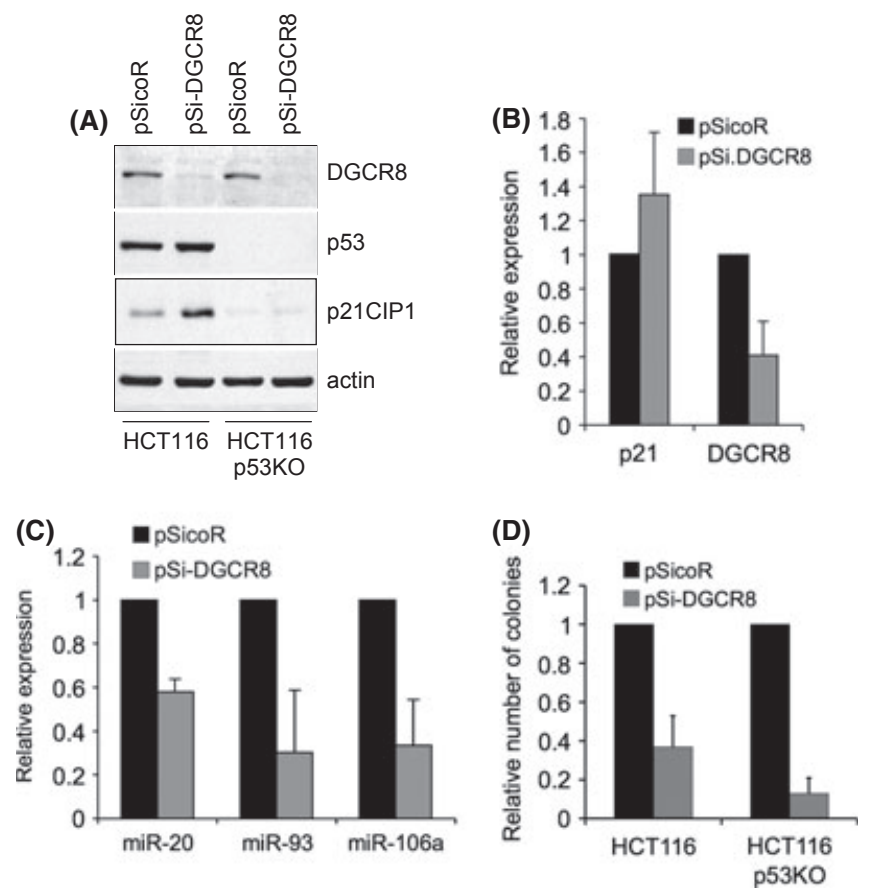

Fig. 5 DGCR8-induced arrest in tumor cell lines. (A) Western blot analysis of the indicated proteins after infection with an sh-DGCR8 vector or empty vector in HCT116 colon carcinoma cells or its p53-null derivative. (B) QPCR of p21CIP1 and DGCR8 in HCT-116 cells expressing sh-DGCR8, relative to cells with empty vector. (C) QPCR of the indicated mature miRNAs in sh-DGCR8 HCT-116 cells relative to vector cells. $(B, C)$ show average and standard deviation from at least two experiments. (D) Colony formation ability of HCT116 or HCT116-p53 KO cells after expression of sh-DGCR8. The number of colonies was normalized to the colonies obtained in each cell line expressing empty vector. The average and standard deviation from three experiments are shown. locus in mice leads to embryonic lethality (Wang et al., 2007; Stark et al., 2008) and defects in proliferation and differentiation in embryonic stem cells (Wang et al., 2007, 2008; Stark et al., 2008). Similarly, specific ablation of DGCR8 in diverse adult mouse tissues results in severe proliferation and differentiation defects in the affected organs (Yi et al., 2009; Fenelon et al., 2011; Chen et al., 2012). Our data suggest that the arrest triggered by DGCR8 depletion is relayed mainly by the direct action of specific miRNAs on the expression of senescence regulators, although some contribution of alternative pathways remains possible. In particular, we have identified a set of miRNAs that are potential mediators of the senescence arrest, which could act, at least in part, by direct targeting of the cell cycle and senescence mediator p21CIP1, which is consistently upregulated in different shDGCR8-cells, albeit to varying extents. However, our results also suggest that additional factors must be involved. For instance, p2 1 is not essential for the arrest, at least in murine fibroblasts. The differential requirement of $p 21$ could be related in part to the relative impact of p21 in senescence in each cell type, as p21-null MEFs can undergo normal replicative or oncogene-induced senescence (Pantoja \& Serrano, 1999), whereas p21 seems to have a stronger impact in human fibroblast senescence (Brown et al., 1997; Borgdorff et al., 2010). In an attempt to identify potential additional mediators of DGCR8-mediated arrest, we have analyzed the expression of cell-cycle regulators in shDGCR8 IMR90 fibroblasts using a QPCR array (Fig. S6). In this way, we have identified a set of genes potentially regulated via miRNAs in ShDGCR8 cells, based on their upregulation relative to control cells and the presence of predicted miRNA-binding sites. The analysis confirmed the upregulation of p21CIP1 at RNA level in shDGCR8 cells, serving as an internal control. This result suggests that several cell-cycle proteins could cooperate with p21CIP1, contributing directly or indirectly to the arrest triggered by shDGCR8; however, further work would be needed to determine the functional relevance of these genes in DGCR8-induced senescence.

Interestingly, the set of miRNAs with reduced expression during DGCR8-mediated arrest are also downregulated during oncogeneinduced senescence (Fig. 4A), replicative senescence (Bonifacio \& Jarstfer, 2010; Marasa et al., 2010; Wang et al., 2011; Faraonio et al., 2012) or DNA-damage induced senescence (Greussing et al., 2013) in different strains of human primary fibroblasts. In addition, some of these miRNAs have been shown to bypass oncogene-induced senescence in human fibroblasts or epithelial cells (Voorhoeve et al., 2006; Borgdorff et al., 2010; Gorospe \& Abdelmohsen, 2011) and are overexpressed in tumors (Ivanovska et al., 2008). Collectively, these results support the notion that downregulation of these specific miRNAs is closely associated to senescence and tumor suppression.

In a recent report, loss of Dicer was shown to lead to premature senescence in murine fibroblasts. In this study, Dicer-induced senescence was relayed by mechanisms different to those shown here, which involved DNA damage and Arf-dependent activation of p53 (Mudhasani et al., 2008). Conflicting recent reports suggest that siRNA-mediated silencing of Dicer can trigger senescent markers in human fibroblasts (Srikantan et al., 2011) but it can also favor bypass of oncogene-induced senescence (Francia et al., 2012). It is well established that Dicer has functions independent from the canonical miRNA machinery, including heterochromatin formation, and production of noncanonical miRNA (Winter et al., 2009). Similarly, Drosha has been reported to play a role in ribosomal RNA processing (Liang \& Crooke, 2011). It is therefore feasible that some phenotypes associated to Dicer or Drosha depletion may reflect miRNA-independent functions, as has been directly shown in some cases (Francia et al., 2012). In contrast, DGCR8 participates exclusively in the processing of canonical miRNAs and, as such, we are confident that our results with inactivation of DGCR8 reflect bona fide 
consequences of miRNA disruption in primary fibroblasts. From a general point of view, our results are consistent with a model where global disruption of the miRNA machinery is associated with activation of an antiproliferative tumor-suppressive barrier in primary cells. In apparent contrast, it has been suggested that miRNA expression is generally downregulated in carcinomas relative to normal tissues (Lu et al., 2005), possibly due to defective miRNA processing (Thomson et al., 2006). However, expression studies of miRNA regulators in cancer have failed to give a consistent picture, with examples of downregulation, overexpression or unaltered expression of key miRNA factors in tumors (Sugito et al., 2006; Muralidhar et al., 2007; Melo et al., 2010). It is well established that specific miRNAs can have opposing functions in tumorigenesis, either as oncogenes, promoting transformation, or as tumor suppressors, preventing transformation. As exemplified in murine embryo stem cells (Melton \& Blelloch, 2010), the balance between miRNA families with opposing roles could determine the cellular outcome of impairment in miRNA function, also in the context of senescence and transformation. Of note, our findings also have implications in the context of tumors, as we show here that the ability to arrest cell cycle in response to disrupted miRNA synthesis is retained in tumor cells, irrespective of their p53 status. While this manuscript was in preparation, similar effects in tumor cell lines have been reported (Peric et al., 2012), in agreement with our work and in contrast to previous reports suggesting the opposite effect (Kumar et al., 2007). In summary, our data show that global miRNA disruption can trigger cellular senescence via deregulation of specific miRNAs and key cell-cycle regulators, highlighting the importance of miRNA-mediated regulation in the control of this tumor-suppressive response.

\section{Experimental procedures}

\section{Cell culture}

IMR90 primary human diploid fibroblasts were obtained from the American Type Culture Collection (ATCC). The p21-knockout and p53knockout MEFs were a kind gift of Manuel Serrano, CNIO, Spain. Earlypassage cells were used for all the experiments. All cells were grown in Dulbecco's Modified Eagles Medium (GIBCO) supplemented with 10\% fetal calf serum and containing antibiotics, at $37^{\circ} \mathrm{C}$ in $5 \% \mathrm{CO}_{2}$.

\section{Retroviral and lentiviral infection}

Retroviral and lentiviral transductions were performed as previously described (Gomez-Cabello et al., 2010; Abad et al., 2011). pRetroSuper mouse Dgcr8, pSicoR human DGCR8, pSicoR human Dicer, pSicoR human Drosha (Kumar et al., 2007), and relevant empty vectors were obtained from Addgene; pRetroSuper human p53 and pRetroSuper human $\mathrm{Rb}$ were a kind gift of Daniel Peeper, NKI, the Netherlands. Ectopic miRNAs were expressed using the retroviral miR-Vec vector.

\section{BrdU incorporation}

Bromodeoxyuridine (BrdU) incorporation was measured essentially as described (Abad et al., 2011). Briefly, infected cells were plated in 8-well glass chamber slides (LabTek, Rochester, NY, USA) at 20000 cells per well. Twenty-four hours later, cells were incubated with $10 \mu \mathrm{m} \mathrm{BrdU}$ for $6 \mathrm{~h}$. BrdU-positive cells were detected by immunofluorescence using an antiBrdU antibody (Megabase Research Products, Lincoln, NE, USA) and DAPI to stain nuclei. At least 200 nuclei were counted to determine the percentage of BrdU-positive cells.

\section{Quantitative RT-PCR}

Total RNA was isolated with Tri-Reagent (Applied Biosystem/Ambion, Carlsbad, CA, USA), as recommended by the manufacturer. CDNA was synthesized using M-MLV reverse transcriptase (Promega, Madison, WI, USA). Quantitative RT-PCR was performed using one microgram of CDNA of each condition, as described (Gomez-Cabello et al., 2010). The primers used are available on request. QPCR of miRNAs was performed using Exiqon or TaqMan probes according to manufacturer's instructions.

\section{Growth assays}

For growth curves, infected cells were seeded in 24-well plates, at 20000 cells per well, in triplicate. At the indicated time points, cells were trypsinized and counted with a Neubauer chamber. For crystal violet staining of plates, cells were plated at low density $\left(5 \times 10^{4}\right.$ cells per $10-\mathrm{cm}$ dish), and at 13 days postselection, they were fixed with formaldehyde and stained with crystal violet. In some experiments, cell number was estimated by staining with crystal violet, followed by solubilization and measurement of absorbance at $595 \mathrm{~nm}$.

\section{UTR luciferase reporter assay}

HEK293T cells were reverse transfected using Polyethylenimine (PEl; Sigma, St. Louis, MO, USA) to individually transfect clones from the miRVec library in a 96-well plate format. A 9:1 ratio of miR-Vec to luciferase reporter construct was used. miR-Vec-Ctrl was used as control vector. A 3:1 ratio of PEI to DNA was used, and after incubation of reagent-DNA complexes for $30 \mathrm{~min}$, cells were added. Firefly and Renilla luciferase activities were measured using the Dual-Luciferase Reporter Assay system (Promega), $48 \mathrm{~h}$ after transfection.

\section{Colony formation assay}

Cells were seeded at a density of 8000 per well in 6-well plates in triplicate. After 10 days, cells were fixed with formaldehyde and stained with Giemsa stain solution, and colonies were counted.

\section{Western blot}

Preparation of protein lysates and immunoblot was carried out as previously described (Palmero et al., 2002). The following antibodies were used: DGCR8 (10996-1-AP; PTG Lab, Manchester, UK), Ras (OP-40; Calbiochem, La Jolla, CA, USA), ERK (sc-92; Santa Cruz Biotechnology Inc., Santa Cruz, CA, USA), Phospho-ERK (sc-7383; Santa Cruz Biotechnology Inc.), human p53 (sc-126; Santa Cruz Biotechnology Inc.), mouse p53 (CM5; Novocastra, Wetzlar, Germany), p21CIP1 (sc-397; Santa Cruz Biotechnology Inc.), p19Arf (54-75; gift from David Parry, DNAX, USA), and Actin (Ac-15; Sigma).

\section{Senescence-associated beta-galactosidase assay}

Infected cells were plated at low density, fixed, and stained for senescence-associated beta-galactosidase activity as previously described (Dimri et al., 1995).

\section{Immunofluorescence}

Immunofluorescence was performed as described (Abad et al., 2011). For quantification of p21-positive cells, immunofluorescence was 
performed with an antibody against p21CIP1, using an IN Cell Analyzer 1000 (GE Healthcare, Piscataway, NJ, USA), followed by image processing and quantification with the IN Cell Investigators software (GE Healthcare).

\section{Senescence-associated heterochromatin foci}

MEFs and IMR90 cells were stained with DAPI and analyzed by microscopy to identify cells with heterochromatin foci, using at least 100 nuclei per each condition.

\section{Acknowledgments}

This work was supported by grants SAF2009-09031 and SAF201232117 from the Spanish Government to IP, and core support from MRC and grants from MRCT, CRUK, AICR, and the EMBO Young Investigator Programme to JG. We are grateful to Manuel Serrano and Daniel Peeper for reagents and advice, and to Pablo Huertas for help with DDR assays.

\section{Author contributions}

DG-C, IA, DG, HK, YT and KT performed the experiments, analyzed the data and helped with the manuscript, JG and IP designed and supervised experiments and DG-C, JG and IP wrote the manuscript.

\section{Conflict of interest}

The authors declare that no conflict of interest exists.

\section{References}

Abad M, Moreno A, Palacios A, Narita M, Blanco F, Moreno-Bueno G, Palmero (2011) The tumor suppressor ING1 contributes to epigenetic control of cellular senescence. Aging Cell 10, 158-171.

Bonifacio LN, Jarstfer MB (2010) miRNA profile associated with replicative senescence, extended cell culture, and ectopic telomerase expression in human foreskin fibroblasts. PLOS ONE 5, e12519.

Borgdorff V, Lleonart ME, Bishop CL, Fessart D, Bergin AH, Overhoff MG, Beach DH (2010) Multiple microRNAs rescue from Ras-induced senescence by inhibiting p21(Waf1/Cip1). Oncogene 29, 2262-2271.

Brown JP, Wei W, Sedivy JM (1997) Bypass of senescence after disruption of p21CIP1/ WAF1 gene in normal diploid human fibroblasts. Science 277, 831-834.

Chen Z, Wu J, Yang C, Fan P, Balazs L, Jiao Y, Lu M, Gu W, Li C, Pfeffer LM, Tigyi G, Yue J (2012) DiGeorge syndrome critical region 8(DGCR8) -mediated miRNA biogenesis is essential for vascular smooth muscle cell development in mice. J. Biol. Chem. 287, 19018-19028.

Collado M, Serrano M (2010) Senescence in tumours: evidence from mice and humans. Nat. Rev. Cancer 10, 51-57.

Di Micco R, Fumagalli M, Cicalese A, Piccinin S, Gasparini P, Luise C, Schurra C, Garre M, Nuciforo PG, Bensimon A, Maestro R, Pelicci PG, d'Adda di Fagagna F (2006) Oncogene-induced senescence is a DNA damage response triggered by DNA hyper-replication. Nature 444, 638-642.

Dimri GP, Lee X, Basile G, Acosta M, Scott G, Roskelley C, Medrano EE, Linskens M, Rubelj I, Pereira-Smith O, Peacocket M, Campisi J. (1995) A biomarker that identifies senescent human cells in culture and in aging skin in vivo. Proc. Natl Acad. Sci. U.S.A. 92, 9363-9367.

Esteller M (2011) Non-coding RNAs in human disease. Nat. Rev. Genet. 12, 861-874.

Faraonio R, Salerno P, Passaro F, Sedia C, laccio A, Bellelli R, Nappi TC, Comegna M, Romano S, Salvatore G, Santoro M, Cimino F (2012) A set of miRNAs participates in the cellular senescence program in human diploid fibroblasts. Cell Death Differ. 19, 713-721.

Fenelon K, Mukai J, Xu B, Hsu PK, Drew LJ, Karayiorgou M, Fischbach GD, Macdermott AB, Gogos JA (2011) Deficiency of Dgcr8, a gene disrupted by the $22 q 11.2$ microdeletion, results in altered short-term plasticity in the prefrontal cortex. Proc. Natl Acad. Sci. U.S.A. 108, 4447-4452.
Francia S, Michelini F, Saxena A, Tang D, de Hoon M, Anelli V, Mione M, Carninc $P$, d'Adda di Fagagna $F$ (2012) Site-specific DICER and DROSHA RNA products control the DNA-damage response. Nature 488, 231-235.

Gomez-Cabello D, Callejas S, Benguria A, Moreno A, Alonso J, Palmero I (2010) Regulation of the microRNA processor DGCR8 by the tumor suppressor ING1. Cancer Res. 70, 1866-1874.

Gorospe M, Abdelmohsen K (2011) MicroRegulators come of age in senescence. Trends Genet. 27, 233-241.

Greussing R, Hackl M, Charoentong P, Pauck A, Monteforte R, Cavinato M, Hofer E, Scheideler M, Neuhaus M, Micutkova L, Mueck C, Trajanoski Z, Grillari J, Jansen-Dürr P (2013) Identification of microRNA-mRNA functional interactions in UVB-induced senescence of human diploid fibroblasts. BMC Genomics 14, 224

Guo H, Ingolia NT, Weissman JS, Bartel DP (2010) Mammalian microRNAs predominantly act to decrease target mRNA levels. Nature 466, 835-840.

Ivanovska I, Ball AS, Diaz RL, Magnus JF, Kibukawa M, Schelter JM, Kobayashi SV, Lim L, Burchard J, Jackson AL, Linsley PS, Cleary MA (2008) MicroRNAs in the miR-106b family regulate p21/CDKN1A and promote cell cycle progression. Mol. Cell. Biol. 28, 2167-2174.

Johnson SM, Grosshans $H$, Shingara J, Byrom M, Jarvis R, Cheng A, Labourier $E_{\text {, }}$ Reinert KL, Brown D, Slack FJ (2005) RAS is regulated by the let-7 microRNA family. Cell 120, 635-647.

Kuilman T, Michaloglou C, Mooi WJ, Peeper DS (2010) The essence of senescence. Genes Dev. 24, 2463-2479.

Kumar MS, Lu J, Mercer KL, Golub TR, Jacks T (2007) Impaired microRNA processing enhances cellular transformation and tumorigenesis. Nat. Genet. 39, 673-677.

Liang XH, Crooke ST (2011) Depletion of key protein components of the RISC pathway impairs pre-ribosomal RNA processing. Nucleic Acids Res. 39, 4875-4889.

Lu J, Getz G, Miska EA, Alvarez-Saavedra E, Lamb J, Peck D, Sweet-Cordero A, Ebert BL, Mak RH, Ferrando AA, Downing JR, Jacks T, Horvitz HR, Golub TR (2005) MicroRNA expression profiles classify human cancers. Nature 435, 834-838.

Lujambio A, Lowe SW (2012) The microcosmos of cancer. Nature 482, 347-355.

Marasa BS, Srikantan S, Martindale JL, Kim MM, Lee EK, Gorospe M, Abdelmohsen $\mathrm{K}$ (2010) MicroRNA profiling in human diploid fibroblasts uncovers miR-519 role in replicative senescence. Aging 2, 333-343.

Melo SA, Moutinho C, Ropero S, Calin GA, Rossi S, Spizzo R, Fernandez AF, Davalos V, Villanueva A, Montoya G, Yamamoto H, Schwartz S Jr, Esteller M (2010) A genetic defect in exportin-5 traps precursor microRNAs in the nucleus of cancer cells. Cancer Cell 18, 303-315.

Melton C, Blelloch R (2010) MicroRNA Regulation of Embryonic Stem Cell Self-Renewal and Differentiation. Adv. Exp. Med. Biol. 695, 105-117.

Mudhasani R, Zhu Z, Hutvagner G, Eischen CM, Lyle S, Hall LL, Lawrence JB, Imbalzano AN, Jones SN (2008) Loss of miRNA biogenesis induces p19Arf-p53 signaling and senescence in primary cells. J. Cell Biol. 181, 1055-1063.

Muralidhar B, Goldstein LD, Ng G, Winder DM, Palmer RD, Gooding EL, Barbosa-Morais NL, Mukherjee G, Thorne NP, Roberts I, Pett MR, Coleman N (2007) Global microRNA profiles in cervical squamous cell carcinoma depend on Drosha expression levels. J. Pathol. 212, 368-377.

Palmero I, Pantoja C, Serrano M (1998) p19ARF links the tumour suppressor p53 to Ras. Nature 395, 125-126.

Palmero I, Murga M, Zubiaga A, Serrano M (2002) Activation of ARF by oncogenic stress in mouse fibroblasts is independent of E2F1 and E2F2. Oncogene 21 2939-2947

Pantoja C, Serrano M (1999) Murine fibroblasts lacking p21 undergo senescence and are resistant to transformation by oncogenic Ras. Oncogene 18, 4974-4982.

Peric D, Chvalova K, Rousselet G (2012) Identification of microprocessor-dependent cancer cells allows screening for growth-sustaining micro-RNAs. Oncogene 31, 2039-2048.

Serrano M, Lin AW, McCurrach ME, Beach D, Lowe SW (1997) Oncogenic ras provokes premature cell senescence associated with accumulation of p53 and p16INK4a. Cell 88, 593-602.

Srikantan S, Gorospe M, Abdelmohsen K (2011) Senescence-associated microRNAs linked to tumorigenesis. Cell Cycle 10, 3211-3212.

Stark KL, Xu B, Bagchi A, Lai WS, Liu H, Hsu R, Wan X, Pavlidis P, Mills AA, Karayiorgou M, Gogos JA (2008) Altered brain microRNA biogenesis contributes to phenotypic deficits in a 22q11-deletion mouse model. Nat. Genet. 40, 751-760.

Sugito N, Ishiguro $H$, Kuwabara $Y$, Kimura M, Mitsui A, Kurehara $H$, Ando T, Mori R, Takashima N, Ogawa R, Fujii Y (2006) RNASEN regulates cell proliferation and affects survival in esophageal cancer patients. Clin. Cancer Res. 12, 7322-7328.

Thomson JM, Newman M, Parker JS, Morin-Kensicki EM, Wright T, Hammond SM (2006) Extensive post-transcriptional regulation of microRNAs and its implications for cancer. Genes Dev. 20, 2202-2207.

Voorhoeve PM, le Sage C, Schrier M, Gillis AJ, Stoop H, Nagel R, Liu YP, van Duijse J, Drost J, Griekspoor A, Zlotorynski E, Yabuta N, De Vita G, Nojima H, Looijenga 
LH, Agami R (2006) A genetic screen implicates miRNA-372 and miRNA-373 as oncogenes in testicular germ cell tumors. Cell 124, 1169-1181.

Wang Y, Medvid R, Melton C, Jaenisch R, Blelloch R (2007) DGCR8 is essential for microRNA biogenesis and silencing of embryonic stem cell self-renewal. Nat. Genet. 39, 380-385.

Wang Y, Baskerville S, Shenoy A, Babiarz JE, Baehner L, Blelloch R (2008) Embryonic stem cell-specific microRNAs regulate the G1-S transition and promote rapid proliferation. Nat. Genet. 40, 1478-1483.

Wang M, Cheng Z, Tian T, Chen J, Dou F, Guo M, Cong YS (2011) Differential expression of oncogenic miRNAs in proliferating and senescent human fibroblasts. Mol. Cell. Biochem. 352, 271-279.

Winter J, Jung S, Keller S, Gregory RI, Diederichs S (2009) Many roads to maturity: microRNA biogenesis pathways and their regulation. Nat. Cell Biol. 11, 228-234.

Wu S, Huang S, Ding J, Zhao Y, Liang L, Liu T, Zhan R, He X (2010) Multiple microRNAs modulate p21Cip1/Waf1 expression by directly targeting its $3^{\prime}$ untranslated region. Oncogene 29, 2302-2308.

Yi R, Pasolli HA, Landthaler M, Hafner M, Ojo T, Sheridan R, Sander C, O'Carroll D, Stoffel M, Tuschl T, Fuchs E (2009) DGCR8-dependent microRNA biogenesis is essential for skin development. Proc. Natl Acad. Sci. U.S.A. 106, 498-502.

Zindy F, Quelle DE, Roussel MF, Sherr CJ (1997) Expression of the p16INK4a tumor suppressor versus other INK4 family members during mouse development and aging. Oncogene 15, 203-211.

\section{Supporting Information}

Additional Supporting Information may be found in the online version of this article at the publisher's web-site:

Fig. S1 Antiproliferative effect of abrogation of DGCR8 in fibroblasts. (A) QPCR analysis of the silencing efficiency of the indicated shRNA vectors, transcript levels for Dicer, Drosha or DGCR8 was normalized to the levels in empty vector-infected cells. (B) Long term proliferation of IMR-90 human fibroblasts retrovirally infected with two shRNAs against DGCR8. (C) Crystal violet staining of IMR-90 human fibroblasts retrovirally infected as in A. (D) Growth curve of IMR-90 human fibroblasts infected with two independent shRNAs against Dicer or Drosha, or an empty vector.

Fig. S2. (A) Western blot analysis of the indicated proteins in IMR90 fibroblasts, after infection with shRNA vectors. (B) Western blot analysis of the indicated proteins in MEF of the indicated genotypes, after infection with shRNA vectors. Erk, total Erk1/2 protein; P-Erk, phosphorylated Erk1/2.

Fig. S3 (A) Representative images of DAPI staining of nuclei from MEFs of the indicated genotype, top, or IMR-90 human fibroblasts, bottom, expressing the indicated shRNA vectors. (B) Percentage of nuclei positive for gammaH2AX staining in IMR-90 and BJ fibroblasts. BJ fibroblasts irradiated with 10 Gy of gamma irradiation are shown as a positive control.

Fig. S4 Schematic representation of target sequences for the indicated miRNAs in the $3^{\prime} U T R$ region of human p21CIP1, showing their complementarity to seed sequences of the miRNAs.

Fig. S5 (A) Relative cell number estimated by crystal violet staining of HCT116 and HCT-116 p53KO cells after silencing of DGCR8. (B) Western blot analysis of the indicated proteins in U2OS or 293T cells, after infection and selection (U2OS) or transient transfection (293T) with the indicated shRNA vectors.

Fig. S6 Cell-cycle regulators with differential expression in shDGCR8 IMR90 cells. 